\title{
Correction to: Hope for male fecundity: clinically insignificant changes in semen parameters over 10 years at a single clinic while assessing an infertility population
}

\author{
Ido Feferkorn ${ }^{1}$ (1) Guy Shrem ${ }^{1,2} \cdot$ Liat Azani $^{3} \cdot$ Weon-Young Son ${ }^{1} \cdot$ Tamar Nehushtan $^{3} \cdot$ Mali Salmon-Divon $^{3,4}$. \\ Michael Haim Dahan ${ }^{1}$
}

Published online: 13 September 2021

○) Springer Science+Business Media, LLC, part of Springer Nature 2021

\section{Correction to: Journal of Assisted Reproduction and Genetics} https://doi.org/10.1007/s10815-021-02298-8

The original article unfortunately was published with an error. Early versions of some of the figures were used that contained several issues.

The original article has been corrected.

Publisher's note Springer Nature remains neutral with regard to jurisdictional claims in published maps and institutional affiliations.

The original article can be found online at https://doi.org/10.1007/ s10815-021-02298-8.

Ido Feferkorn

ido.feferkorn@mail.mcgill.ca

1 Division of Reproductive Endocrinology and Infertility, McGill University Health Care Center, 888 Boul. de Maisonneuve E \#200, Montréal, QC H2L 4S8, Canada

2 IVF Unit, Department of Obstetrics and Gynecology, Kaplan Medical Center, 1 Derech Pasternak, Rehovot, Israel

3 Department of Molecular Biology, Ariel University, 65 Ramat HaGolan St, Ariel, Israel

4 Adelson School of Medicine, Ariel University, 65 Ramat HaGolan St, Ariel, Israel 